\title{
Associations with Organ Involvement and Autoantibodies in Systemic Sclerosis: Results from the Canadian Scleroderma Research Group (CSRG)*
}

\author{
Vikram Tangri ${ }^{1}$, Carly Hewson ${ }^{1}$, Murray Baron ${ }^{2}$, A. Bonner ${ }^{3}$, Marvin Fritzler ${ }^{4}$, Janet E. Pope ${ }^{1}$ \\ ${ }^{1}$ University of Western Ontario, London, Canada; ${ }^{2} \mathrm{McGill}$ University, Montreal, Canada; ${ }^{3}$ McMaster University, Hamilton, Canada; \\ ${ }^{4}$ University of Calgary, Calgary, Canada. \\ Email: janet.pope@sjhc.london.on.ca
}

Received March $6^{\text {th }}, 2013$; revised April $7^{\text {th }}, 2013$; accepted April 14 ${ }^{\text {th }}, 2013$

Copyright (C) 2013 Vikram Tangri et al. This is an open access article distributed under the Creative Commons Attribution License, which permits unrestricted use, distribution, and reproduction in any medium, provided the original work is properly cited.

\begin{abstract}
Objective: Serum from SSc patients was analyzed centrally to determine ANA patterns and extractable nuclear antigens (ENAs) between $\mathrm{lcSSc}$ and dcSSc and associations with organ involvement. Methods: 1145 SSc patients had ANA and ENA analyzed by indirect immunofluorescence on HEp-2 substrate at a screening serum dilution of 1/160. Most ENA antibodies [Sm. U1-RNP, Ro52, SS-A/Ro60, topoisomeraseI (Topo1), SS-B/La, chromatin, ribosomal P and Jo1] were measured by laser bead immunoassay; and RNA polymerase III (RNAP) by ELISA. Results: ANA was positive in $95 \%$ (same in lcSSc, and dcSSc). Centromere pattern was present in 34\%, speckled 22\%, nucleolar 18\%, homogeneous and speckled (H\&S) 16\%, multiple nuclear dots 6\%. Anti-centromere Ab (ACA) occurred in 46\% of lcSSc and $11 \%$ of dcSSc $(\mathrm{P}=0.0001)$. ENAs that differed between lcSSc and dcSSc subsets were Topol (OR 2.4, $\mathrm{P}=0.0001)$ and RNAP (OR 5.6, $\mathrm{P}<0.0001$ ) more common in dcSSc. Overall, 15\% had positive Topo1; usually with a H\&S pattern (67\%); Topo1 was associated with ILD on CXR (OR 2.3; 95\% CI 1.5 - 3.5) and HRCT (OR 3.8; 95\% CI 1.8 - 8.2). RNAP occurred in $18.5 \%$ (35.4\% in dcSSc vs. $8.9 \%$ in lcSSc). Scleroderma renal crisis (SRC) was 13 times more likely if RNAP positive; $\mathrm{P}=0.0001$. ACA was only weakly associated with $\mathrm{SPAP}>50 \mathrm{mmHg}$ (OR 1.8; 95\% $\mathrm{CI} 1.1-3.0)$. Conclusion: ANA homogeneous pattern alone is rare in SSc; ACA was significantly more common in lcSSc. Many ENAs are equal in lcSSc and dcSSc except RNAP and Topol. RNAP has the highest OR of SRC. Topol is less strongly associated with ILD. Abstract word count: 249, Body word count 1246, Figures 2, Tables 2. Key Messages: 1) 95\% of SSc has a positive ANA and ANA patterns in SSc include centromere, nucleolar, and homogeneous and speckled together; 2) Most ENAs are equal in both dcSSc and lcSSc except anti RNA polymerase III and topoisomerase I; 3) RNA polymerase III has the highest association (odds ratio) with scleroderma renal crisis, topoisomerase I is associated with interstitial lung disease; whereas anticentromere was not associated with elevated pulmonary arterial pressures on echocardiogram.
\end{abstract}

Keywords: Scleroderma; SSc; Systemic Sclerosis; Antibodies; Anticentromere; Topoisomerase I; RNA Polymerase III; Organ Involvement; Scleroderma Renal Crisis; Pulmonary Fibrosis; ILD; ANA; Pulmonary Hypertension

\section{Introduction}

Systemic Sclerosis ( $\mathrm{SSc}$ ) is an autoimmune disorder cau-

${ }^{*}$ CSRG (Canadian Scleroderma Research Group) is: J. Pope, London, Ontario; M. Baron, Montreal, Quebec; J. Markland, Saskatoon, Saskatchewan; D. Robinson, Winnipeg, Manitoba; N. Jones, Edmonton, Alberta; N. Khalidi, Hamilton, Ontario; P. Docherty, Moncton, New Brunswick; E. Kaminska, Hamilton, Ontario; A. Masetto, Sherbrooke, Quebec; E. Sutton, Halifax, Nova Scotia; J-P. Mathieu, Montreal, Quebec; M. Hudson, Montreal, Quebec; S. Ligier, Montreal, Quebec; T. Grodzicky, Montreal, Quebec; S. LeClercq, Calgary, Alberta; C. Thorne, Newmarket, Ontario; G. Gyger, Montreal, Quebec; D. Smith, Ottawa, Ontario; M. Fritzler, Advanced Diagnostics Laboratory, Calgary, Alberta. sing various clinical manifestations related to skin fibrosis and internal organ dysfunction. Limited cutaneous systemic sclerosis (lcSSc) primarily affects the skin of the fingers and hands, while diffuse cutaneous systemic sclerosis $(\mathrm{dcSSc})$ is known to affect skin tissue and organs more extensively [1]. LcSSc is defined as skin involvement distal to the elbows/knees but may still affect the face and neck; whereas dcSSc involves skin that is also proximal to the elbows and/or knees or also truncal [1].

The presence of autoantibodies can be associated with disease pathology and progression. Anti nuclear antibo- 
dies (ANA) are found in over $90 \%$ of patients with SSc [2]. The type of ANA is related to reactions against specific components of the cell, and is associated with certain clinical manifestations of disease. Positivity for one type of ANA tends to remain throughout the course of disease, and each type is usually exclusive of the others [3]. Types include anti centromere antibody (ACA), anti-topoisomeraseI (anti-topo I) and anti RNA polymerase III (RNAP).

Thus, antibody profiles are necessary in the evaluation of patients with SSc. In this study, the Canadian Scleroderma Research Group (CSRG) data bank was utilized to determine the distribution of autoantibodies in patients with $\mathrm{lcSSc}$ and dcSSc. The purpose of this study was to especially study the ANA patterns in SSc and study the prevalence of extractable nuclear antigens (ENAs) in SSc such as SSA, SSB and Jo1.

\section{Methods}

Sera from 1145 scleroderma patients registered with CSRG were tested for autoantibody positivity by indirect immunofluorescence on HEp-2 substrate (Immuno Concepts, Sacramento, CA) at a screening serum dilution of $1 / 160$. Screening for anti extractable nuclear antibody (ENA) were carried out by addressable laser bead immunoassay (INOVA Diagnostics, San Diego, CA) to detect Sm. U1-RNP, Ro52, SS-A/Ro60, topoisomeraseI (Topo1),
SS-B/La, chromatin, ribosomal $\mathrm{P}$ and Jo1. Positivity for RNA polymerase III (RNAP) was tested by ELISA (INOVA Diagnostics).

Results were then analyzed by percentage of patients with dcSSc and lcSSc having positivity for these antibodies. P-values and odds ratios were calculated. Rates of SSc renal crisis were obtained from CSRG data for both lcSSc and dcSSc groups. Interstitial lung disease was defined in several ways to determine the strength of antibody associations including changes on chest radiographs, high resolution CT scans and pulmonary function tests with a forced vital capacity of $<70 \%$ predicted in the absence of airways obstruction. Correlations were measured between the systolic pulmonary artery pressure on echocardiogram (sPAP in $\mathrm{mmHg}$ ) and some antibodies.

\section{Results}

There were 1145 patients with scleroderma who were included in this study; 647 of these patients had lcSSc (62\%) while 396 patients had dcSSc (38\%). There was a positive ANA in $95 \%$.

Overall, in the ANA, a centromere pattern was detected in one third of patients. Patterns were $22 \%$ speckled, $18 \%$ nucleolar, $16 \%$ homogeneous and speckled (H\&S), $6 \%$ multiple nuclear dots (i.e. NSp1), 1\% cytoplasmic speckled, $0.5 \%$ homogeneous alone and $0.5 \%$ nuclear membrane. Figure 1 shows the distribution of patterns of

\section{Serum ii05 Distribution in all ANA Positive Patients}

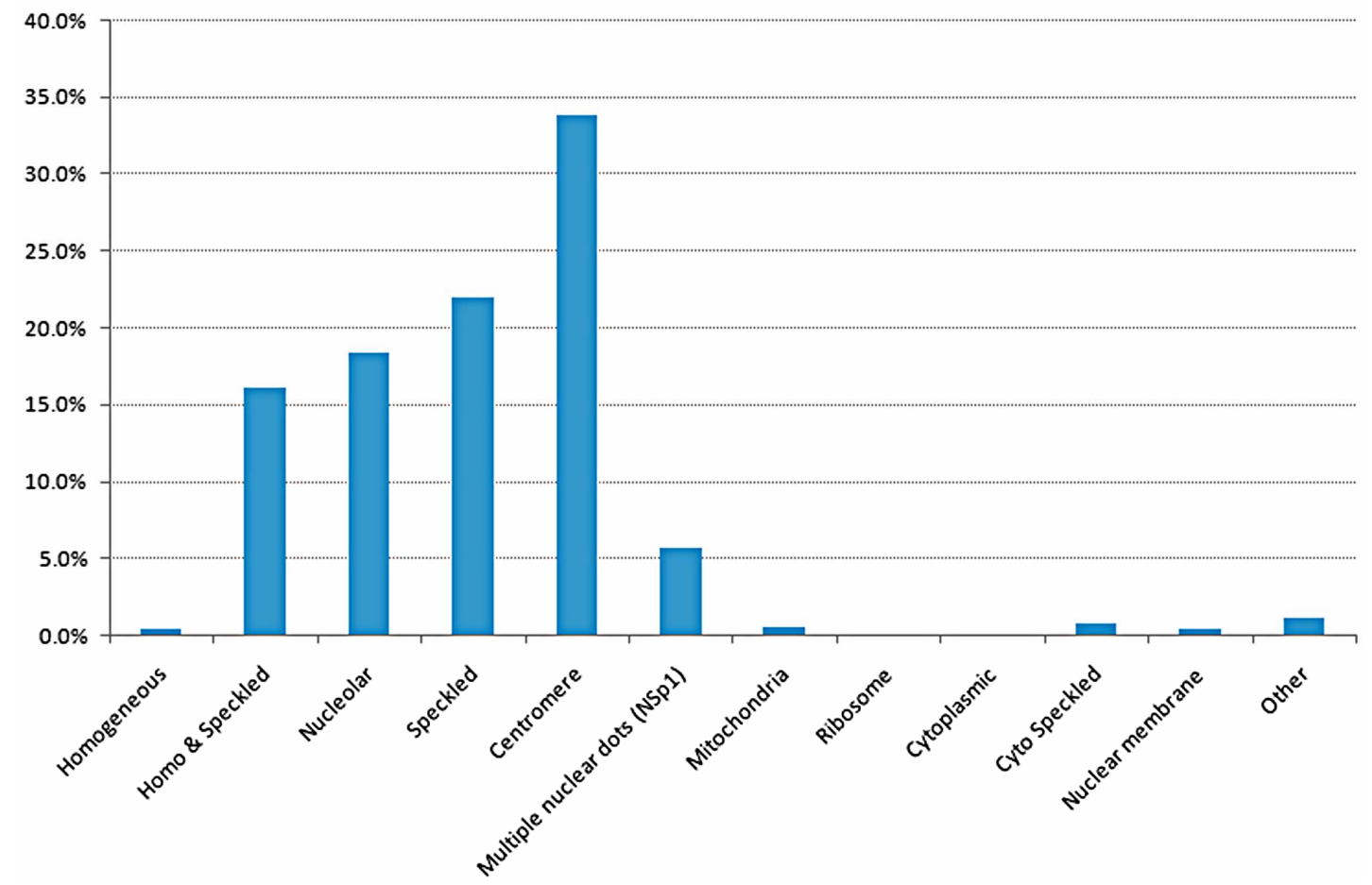

Figure 1. Proportion of ANA positive SSc patients from the CSRG in each ANA pattern. 
positive ANAs within CSRG SSc patients.

ACA was positive in a third of patients; $46 \%$ of $1 \mathrm{cSSc}$ patients and $11 \%$ of deSSc $(\mathrm{P}=0.0001)$. Table 1 shows

Table 1. Baseline characteristics of the CSRG patients.

\begin{tabular}{|c|c|c|c|}
\hline & Total & Diffuse & Limited \\
\hline Number (\%) & 1145 & $396(38 \%)$ & $647(62 \%)$ \\
\hline Age at SSc onset (SD) & $55.4(12.1)$ & $52.6(12.3)$ & $57.1(11.8)$ \\
\hline Gender \% female & $86.0 \%$ & $81.0 \%$ & $89.0 \%$ \\
\hline $\begin{array}{l}\text { Disease Duration } \\
\text { (yrs) (SD) since 1st } \\
\text { non-RP symptom }\end{array}$ & $14.7(12.3)$ & $10.9(10.7)$ & $17.0(12.7)$ \\
\hline ILD on CXR & $22.7 \%$ & $28.2 \%$ & $20.3 \%$ \\
\hline $\begin{array}{l}\text { ILD on HRCT (in } \\
\text { those who had HRCT } \\
\mathrm{N}=\mathbf{2 9 0} \text { ) }\end{array}$ & $62.4 \%$ & $73.0 \%$ & $57.7 \%$ \\
\hline $\begin{array}{l}\text { ILD on PFT } \\
(\text { FVC }<70 \%)\end{array}$ & $13.2 \%$ & $17.8 \%$ & $10.4 \%$ \\
\hline sPAP $>50$ mmHg & $10.0 \%$ & $10.5 \%$ & $9.9 \%$ \\
\hline sPAP $>40 \mathrm{mmHg}$ & $25.7 \%$ & $28.1 \%$ & $25.2 \%$ \\
\hline $\begin{array}{l}\text { Scleroderma Renal } \\
\text { Crisis ever }\end{array}$ & $4.3 \%$ & $8.4 \%$ & $1.9 \%$ \\
\hline Sjogren's/Sicca & $31.0 \%$ & $31.1 \%$ & $30.5 \%$ \\
\hline $\begin{array}{l}\text { Raynaud's } \\
\text { Phenomenon }\end{array}$ & $97.2 \%$ & $96.9 \%$ & $98.1 \%$ \\
\hline SLE & $3.1 \%$ & $2.8 \%$ & $3.4 \%$ \\
\hline $\begin{array}{l}\text { Myositis (elevated } \\
\text { CK and any prox. } \\
\text { musc. weakness) }\end{array}$ & $1.4 \%$ & $2.3 \%$ & $0.9 \%$ \\
\hline $\begin{array}{l}\text { Inflammatory } \\
\text { Arthritis } \\
\text { ( } \mathrm{SJC} \geq \mathbf{4} \text { or } \mathrm{TJC} \geq 4 \text { ) }\end{array}$ & $8.6 \%$ & $10.2 \%$ & $7.6 \%$ \\
\hline$+\mathbf{A N A}$ & $95.0 \%$ & $95.0 \%$ & $95.1 \%$ \\
\hline +ENA & $59.6 \%$ & $70.0 \%$ & $54.5 \%$ \\
\hline+ Centromere & $34.6 \%$ & $11.3 \%{ }^{*}$ & $46.2 \%{ }^{*}$ \\
\hline +Topo1/Scl70 & $15.0 \%$ & $23.4 \%{ }^{*}$ & $11.3 \%{ }^{*}$ \\
\hline +RNApol3 & $18.5 \%$ & $35.4 \%{ }^{*}$ & $8.9 \% *$ \\
\hline+ anti-RNP & $5.9 \%$ & $5.5 \%$ & $6.1 \%$ \\
\hline+ anti-Smith & $3.8 \%$ & $2.5 \%$ & $4.9 \%$ \\
\hline$+\mathbf{P m} /$ Scl & $9.2 \%$ & $10.8 \%$ & $8.2 \%$ \\
\hline +SSA/Ro60 & $9.2 \%$ & $8.8 \%$ & $9.4 \%$ \\
\hline$+\operatorname{Ro52}$ & $19.4 \%$ & $18.2 \%$ & $20.1 \%$ \\
\hline$+\mathrm{SSB} / \mathrm{La}$ & $3.2 \%$ & $3.3 \%$ & $3.3 \%$ \\
\hline$+\mathbf{J o 1}$ & $0.6 \%$ & $0.3 \%$ & $0.9 \%$ \\
\hline +Chromatin & $4.4 \%$ & $6.1 \%$ & $3.7 \%$ \\
\hline +Ribosomal P & $1.4 \%$ & $1.1 \%$ & $1.6 \%$ \\
\hline
\end{tabular}

Data are given as \% or mean (SD) where appropriate. Jo1 is very low in both groups. Topol and RNAPol 3 are more common in dcSSc. ${ }^{*} \mathrm{P}<0.0001$. sPAP is systolic pulmonary artery pressure on echocardiogram. the characteristics of the CSRG patients including the lcSSc and dcSSc subsets. Figure 2 demonstrates the distribution of ENA among patients with lcSSc and dcSSc. TopoI was positive in $15 \%$ overall; twice as frequently in dcSSc than lcSSc. Most of Topo1 were associated with an H\&S pattern (67\%). It was more likely for RNA polymerase 3 (RNAP) to be found in $\operatorname{dcSSc}(35 \%)$ than lcSSc (9\%). As expected, both Topol (OR 2.4, P = $0.0001)$ and RNAP (OR 5.6, P < 0.0001) were far more common in dcSSc versus lcSSc; whereas the likelihood of a positive ACA in dcSSc vs. lcSSc was 0.15; $\mathrm{P}<0001$. The frequencies of other ENAs were similar between both SSc groups. Ro52 occurred most often in centromere, speckled, nucleolar, and H\&S patterns. Jo1 occurred in only 5 patients $(0.6 \%)$ and was always associated with a centromere pattern. The frequency was too low so we did not determine if it was associated with ILD in SSc. Overall just over $4 \%$ of the cohort had scleroderma renal crisis (SRC) (9\% in dcSSc and $2 \%$ in lcSSc). RNAP had a very high chance of scleroderma renal crisis and topol had an association with ILD as measured by CXR, high resolution $\mathrm{CT}$ scan of the chest (HRCT) and restrictive pulmonary function tests (PFTs) but the odds ratio was not as high as what was found for RNAP in SRC. Other ENA frequencies are found in Table 2 with associated $\mathrm{P}$ values and odds ratios. ACA was only weakly associated with elevated systolic pulmonary artery pressure (sPAP > $50 \mathrm{mmHg}$ ).

\section{Discussion}

Autoantibodies are important in the assessment of systemic sclerosis. Certain antibody profiles have been associated with specific clinical subsets of patients, $1 \mathrm{cSSc}$ and dcSSc.

Clinical subsets and antibodies have been found to be variable based on genetic background. In the US, studies have shown an $\mathrm{lcSSc}$ to dcSSc ratio of 1.5:1 [4]. In England, there has been an even higher predominance of

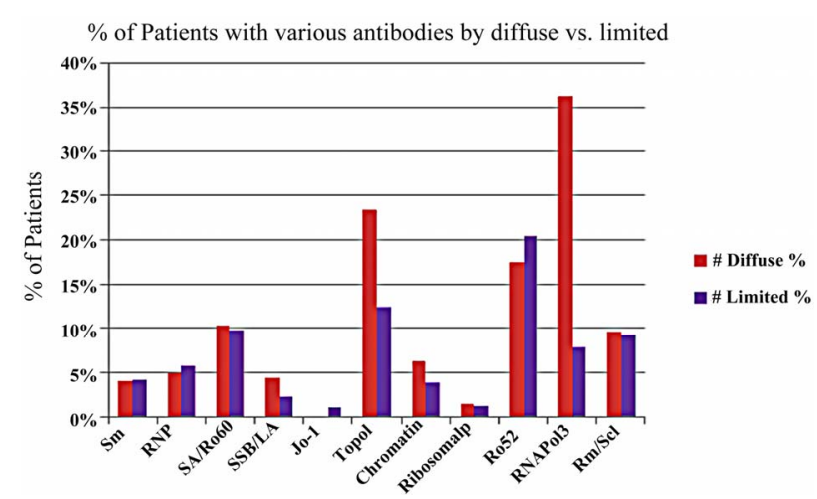

Figure 2. Proportion of patients with varous ENA autoantibodies in SSc patients from the CSRG database. 
Table 2. Correlation coefficients and odds ratios for ENA antibodies and SSc features.

\begin{tabular}{|c|c|c|c|c|}
\hline $\mathrm{N}=1145$ & Spearman Rho & P Value & Odds Ratio & $95 \%$ CIs \\
\hline FVC $<70$ and Topo1/ Scl70 & 0.081 & 0.017 & 1.804 & $1.104-2.947$ \\
\hline ILD on CXR and Topo1/Scl70 & 0.143 & 0.01 & 2.320 & $1.530-3.518$ \\
\hline $\begin{array}{l}\text { ILD on HRCT and Topo1/Scl70 } \\
(N=270)\end{array}$ & 0.220 & 0.01 & 3.823 & $1.781-8.205$ \\
\hline SRC and RNApol3 & 0.309 & 0.01 & 13.191 & $6.364-27.338$ \\
\hline sPAP $>50$ and centromere & 0.089 & 0.027 & 1.799 & $1.062-3.048$ \\
\hline sPAP $>50$ and Topo1/Scl70 & -0.058 & 0.153 & 0.508 & $0.198-1.306$ \\
\hline sPAP $>50$ and $R N P$ & -0.031 & 0.437 & 0.566 & $0.132-2.422$ \\
\hline sPAP $>50$ and RNApol3 & -0.017 & 0.717 & 0.862 & $0.388-1.918$ \\
\hline sPAP $>50$ and PMScl & -0.024 & 0.606 & 0.726 & $0.215-2.454$ \\
\hline SPAP $>50$ and Ro52 & 0.080 & 0.047 & 1.803 & $1.001-3.248$ \\
\hline sPAP > 50 and Ro60 & 0.000 & 0.997 & 0.998 & $0.381-2.616$ \\
\hline Sjogren's and Ro52 & 0.054 & 0.083 & 1.333 & $0.963-1.847$ \\
\hline Sjogren's and Ro60 & 0.056 & 0.075 & 1.485 & $0.960-2.299$ \\
\hline Sjogren's and La & -0.004 & 0.906 & 0.956 & $0.449-2.032$ \\
\hline Sjogren's and Ro(52 or 60$)+\mathrm{La}$ & 0.025 & 0.421 & 1.388 & $0.623-3.094$ \\
\hline Sjogren's and Ro52 + La & 0.031 & 0.323 & 1.539 & $0.651-3.639$ \\
\hline Sjogren's and Ro60 + La & 0.021 & 0.503 & 1.330 & $0.576-3.072$ \\
\hline SLE and anti-Smith & 0.112 & 0.01 & 5.234 & $1.898-14.439$ \\
\hline $\begin{array}{l}\text { Myositis/myopathy (elevated CK and } \\
\text { any prox. musc. weakness) and PMScl }\end{array}$ & 0.003 & 0.926 & 1.103 & $0.138-8.836$ \\
\hline MD Diagnosed Myositis and PMScl & 0.019 & 0.591 & 1.402 & $0.408-4.819$ \\
\hline Myositis/myopathy and Jo1 & -0.009 & 0.770 & 0 & \\
\hline Myositis/myopathy and RNApol3 & -0.025 & 0.494 & 0.492 & $0.062-3.912$ \\
\hline $\begin{array}{l}\text { Inflammatory Arthritis } \\
\text { (SJC } \geq 1 \text { and } \mathrm{TJC} \geq 1 \text { ) }\end{array}$ & $\begin{array}{l}\text { No significant } \\
\text { correlations/ORs }\end{array}$ & & & \\
\hline FVC $<70$ and Jo1 & 0.017 & 0.624 & 1.725 & $0.191-15.574$ \\
\hline CXR and Jo1 & 0.072 & 0.042 & 5.302 & $0.879-31.980$ \\
\hline HRCT and Jo1 $(\mathrm{N}=270)$ & 0.082 & 0.179 & $\begin{array}{l}\text { unable to calculate } \\
\text { (value in } 2 \times 2 \\
\text { table }=0)\end{array}$ & \\
\hline Limited Ssc and centromere & 0.363 & 0.01 & 6.754 & $4.692-9.722$ \\
\hline Diffuse Ssc and Topo1/Scl70 & 0.16 & 0.01 & 2.39 & $1.676-3.406$ \\
\hline Diffuse SSc and RNApol3 & 0.329 & 0.01 & 5.648 & $3.740-8.528$ \\
\hline No Raynaud's and Ro60 & 0.066 & 0.036 & 2.828 & $1.025-7.802$ \\
\hline
\end{tabular}

Definitions: ILD on CXR—abnormal chest $\mathrm{x}$-ray showing increased interstitial markings (not due to CHF) or evidence of fibrosis; ILD on HRCT-abnormal HRCT showing any degree of ground glass changes or fibrotic interstitial changes or honeycombing; $\mathrm{SPAP}>50$ - systolic pulmonary artery pressure measured on echocardiogram > $50 \mathrm{mmHg}$; Sjogren's - at least 3 symptoms of dryness or 2 symptoms of dryness and 1 sign of dryness on physical exam; Myositis/Myopathy-Elevated CK ( $>200$ females, $>250$ males) and any proximal muscle weakness (motor power $<$ grade 5$)$; MD Diagnosed Myositis-MD diagnosed polymyositis as overlapping condition; Inflammatory Arthritis swollen joint count $\geq 1$ and tender joint count $\geq 1$; SLE—MD diagnosed SLE as overlapping condition.

patients having lcSSc compared to dcSSc [5]. In the present study of Canadian patients, approximately $60 \%$ of the SSc patients had lcSSc.

ACA is frequently found in patients with $\mathrm{SSc}$ and is 
highly specific $(99.8 \%-100 \%)$ for this disease [3]. It is seen more in patients of Caucasian background compared with other ethnicities in the US; studies have demonstrated ACA positivity in $20 \%-30 \%$ of SSc patients [6]. ACA positivity is seen with SSc associated with pulmonary arterial hypertension [7]. ACA positive SSc patients also have an increased likelihood of developing primary billiary cirrhosis [8]. It can also be utilized as a predictor for development of SSc in patients with known Raynaud's phenomenon [9]. ACA positive rates were similar to previous data.

TopoI is another antibody that is commonly seen in SSc; its specificity is $97 \%-100 \%$ [10]. Topol has been found in both lcSSc and dcSSc patients, but is more common in dcSSc. It can be utilized to predict development of cutaneous ulcers and pulmonary fibrosis [7,11,12]. Indeed, TopoI positivity may be associated with a higher risk of renal crisis [12]. It has been positive in $40 \%$ of dcSSc patients and under $10 \%$ of patients with $1 \mathrm{cSSc}$ $[3,13]$. This study showed TopoI positivity in $16.7 \%$ overall; a higher rate was found in dcSSc versus lcSSc. Thus the prevalence of TopoI in the CSRG group was lower than that found in other populations.

RNAP positivity is also highly specific $(98 \%-100 \%)$ for SSc [14]. Sera showing RNAP has been found in $20 \%$ of SSc patients; it is usually associated with dcSSc $[15,16]$. Demonstration of this antibody in patients with SSc is correlated with development of renal crisis [7]. The present study shows a similar rate of RNAP in patients with SSc, with an expected predilection for dcSSc patients. Other autoantibodies such as anti-U3RNP and anti-Th/To are described in SSc but we did not have them performed; which is a limitation.

In the CSRG database, there could have been some misclassification of previous dcSSc as $1 \mathrm{cSSc}$, since proximal involvement of skin may not have been known at cohort entry. This may indeed affect the prevalence rates demonstrated in the study.

$\mathrm{PH}$ associated with ILD had less ACA than PAH in one SSc study implying PAH was increased if ACA postivie [17] but not in another paper [18].

\section{Conclusion}

Some ANA patterns were common in SSc. Homogeneous pattern alone was rare. Although many ENAs had similar prevalence in both $\mathrm{lcSSc}$ and dcSSc, both RNAP and Topol were more common in dcSSc. ACA was more frequent in lcSSc but was not an important association in those with elevated SPAP.

\section{Funding}

The Canadian Scleroderma Research Group (CSRG) is supported financially by the Canadian Institutes of Health Research (CIHR), the Scleroderma Society of Canada, Scleroderma Society of Ontario, Cure Scleroderma Foundation. Some educational grants received from Pfizer Pharmaceuticals and Actelion Canada. Kits for ANA and ENA testing were provided by ImmunoConcepts, Sacramento, CA, and INOVA Diagnostics, San Diego, CA.

\section{REFERENCES}

[1] E. C. LeRoy, C. Black, R. Fleischmajer, et al., "Scleroderma (Systemic Sclerosis): Classification, Subsets, and Pathogenesis," Journal of Rheumatology, Vo1. 15, No. 2, 1988, pp. 202-205.

[2] E. M. Tan, "Antinuclear Antibodies: Diagnostic Markers for Autoimmune Diseases and Probes for Cell Biology," Advances in Immunology, Vol. 44, 1989, pp. 93-151. doi:10.1016/S0065-2776(08)60641-0

[3] J. D. Reveille and D. H. Solomon, "Evidence-Based Guidelines for the Use of Immunologic Tests: Anticentromere, Scl-70, and Nucleolar Antibodies," Arthritis Care \& Research, Vol. 49, No. 3, 2003, pp. 399-412. doi:10.1002/art.11113

[4] M. D. Mayes, J. V. Lacey Jr., J. Beebe-Dimmer, B. W. Gillespie, B. Cooper, T. J. Laing, et al., "Prevalence, Incidence, Survival, and Disease Characteristics of Systemic Sclerosis in a Large US Population," Arthritis \& Rheumatism, Vol. 48, No. 8, 2003, pp. 2246-2255. doi:10.1002/art.11073

[5] R. J. Allcock, I. Forrest, P. A. Corris, P. R. Crook and I. D. Griffiths, "A Study of the Prevalence of Systemic Sclerosis in Northeast England," Rheumatology (Oxford), Vol. 43, No. 5, 2004, pp. 596-602.

[6] J. D. Reveille, M. Fischbach, T. McNearney, et al., "Systemic Sclerosis in 3 US Ethnic Groups: A Comparison of Clinical, Sociodemographic, Serologic, and Immunogenetic Determinants," Seminars in Arthritis and Rheumatism, Vol. 30, No. 5, 2001, pp. 332-346. doi:10.1053/sarh.2001.20268

[7] V. Steen, "Autoantibodies in Sytemic Sclerosis," Seminars in Arthritis and Rheumatism, Vol. 35, No. 1, 2005, pp. 35-42. doi:10.1016/j.semarthrit.2005.03.005

[8] C. Rigamonti, et al., "Clinical Features and Prognosis of Primary Biliary Cirrhosis Associated with Systemic Sclerosis," Gut, Vol. 55, No. 3, 2006, pp. 388-394. doi:10.1136/gut.2005.075002

[9] C. G. Kallenberg, A. A. Wouda, M. H. Hoet and W. J. van Venrooij, "Development of Connective Tissue Disease in Patients Presenting with Raynaud's Phenomenon: A Six-Year Follow-up with Emphasis on the Predictive Value of Antinuclear Antibodies as Detected by Immunoblotting," Annals of Rheumatic Diseases, Vol. 477, No. 8, 1988, pp. 634-641. doi:10.1136/ard.47.8.634

[10] D. Basu and J. D. Reveille, "Anti-scl-70," Autoimmunity, Vol. 38, No. 1, 2005, pp. 65-72 doi: 10.1080/08916930400022947

[11] R. Hesselstrand, A. Scheja, G. Q. Shen, A. Wiik and A. 
Akesson, "The Association of Antinuclear Antibodies with Organ Involvement and Survival in Systemic Sclerosis," Rheumatology (Oxford), Vol. 42, No. 4, 2003, pp. 534-540. doi:10.1093/rheumatology/keg170

[12] U. A. Walker, et al., "Clinical Risk Assessment of Organ Manifestations in Systemic Sclerosis: A Report from the eULAR Scleroderma Trials and Research Group Database," Annals of Rheumatic Diseases, Vol. 66, No. 6, 2007, pp. 754-763. doi:10.1136/ard.2006.062901

[13] G. Spencer-Green, D. Alter and H. G. Welch, "Test Performance in Systemic Sclerosis: Anti-Centromere and Anti-Scl-70 antibodies," The American Journal of Medicine, Vol. 103, No. 3, 1997, pp. 242-248. doi:10.1016/S0002-9343(97)00023-5

[14] M. Kuwana, et al., "Enzyme-Linked Immunosorbent Assay for Detection of Anti-RNA Polymerase III Antibody: Analytical Accuracy and Clinical Associations in Systemic Sclerosis," Arthritis \& Rheumatism, Vol. 52, No. 8, 2005, pp. 2425-2432. doi:10.1002/art.21232

[15] E. J. Cepeda and J. D. Reveille, "Autoantibodies in Systemic Sclerosis and Fibrosing Syndromes: Clinical Indi- cations and Relevance," Current Opinion in Rheumatology, Vol. 16, 2004, pp. 723-732. doi:10.1097/01.bor.0000144760.37777.fa

[16] C. C. Bunn, C. P. Denton, X. Shi-Wen, C. Knight and C. M. Black, "Anti-RNA Polymerases and Other Autoantibody Specificities in Systemic Sclerosis," Rheumatology, Vol. 37, No. 1, 1998, pp. 15-20. doi:10.1093/rheumatology/37.1.15

[17] D. Launay, M. Humbert, A. Berezne, V. Cottin, Y. Allanore, L. J. Couderc, O. Bletry, A. Yaici, P. Y. Hatron, L. Mouthon, J. Le Pavec, P. Clerson and E. Hachulla, "Clinical Characteristics and Survival in Systemic SclerosisRelated Pulmonary Hypertension Associated with Interstitial Lung Disease," Chest, Vol. 140, No. 4, 2011, pp. 1016-1024. doi:10.1378/chest.10-2473

[18] F. Boin, S. Franchini, E. Colantuoni, A. Rosen, F. M. Wigley and L. Casciola-Rosen, "Independent Association of Anti-Beta (2)-Glycoprotein I Antibodies with Macrovascular Disease and Mortality in Scleroderma Patients," Arthritis \& Rheumatism, Vol. 60, No. 8, 2009, pp. 24802489. doi:10.1002/art.24684 